\title{
A COMPARATIVE EVALUATION OF DEXMEDETOMIDINE, PROPOFOL AND MIDAZOLAM FOR INTRAOPERATIVE SEDATION IN REGIONAL ANAESTHESIA
}

\author{
Kiwi Mantan', Anita Pareek2, Rashmi Jain ${ }^{3}$, Anju Meena ${ }^{4}$, Pramila Soni5, Aditi Sharma ${ }^{6}$ \\ ${ }^{1}$ Assistant Professor, Department of Anaesthesia, Sardar Patel Medical College and AG of Hospitals, Bikaner, Rajasthan. \\ 2 Professor, Department of Anaesthesia, Sardar Patel Medical College and AG of Hospitals, Bikaner, Rajasthan. \\ ${ }^{3}$ Senior Registrar, Department of Anaesthesia, Sardar Patel Medical College and AG of Hospitals, Bikaner, Rajasthan. \\ ${ }^{4}$ Senior Registrar, Department of Anaesthesia, Sardar Patel Medical College and AG of Hospitals, Bikaner, Rajasthan. \\ ${ }^{5}$ Assistant Professor, Department of Anaesthesia, Sardar Patel Medical College and AG of Hospitals, Bikaner, Rajasthan. \\ ${ }^{6}$ Intership Trainee, Lady Hardinge, MC New Delhi.
}

ABSTRACT

\section{BACKGROUND}

Sedation has been shown to increase patient's satisfaction during regional anaesthesia and may be considered as a mean to increase the patient's acceptance of regional anaesthetic techniques. The aim of the study is to compare dexmedetomidine, propofol and midazolam for intra- and post-operative sedation, haemodynamics and complications.

\section{MATERIALS AND METHODS}

The study was conducted on 80 patients in the Department of Anaesthesiology, Sardar Patel Medical College after taking permission from Institution Research Board. Both male and female patients ranging between the age group of 20 - 60 years belonging to ASA grades I and II, scheduled for elective lower limb or lower abdominal surgical procedure under regional anaesthesia were included. The patients were divided into 4 groups of 20 patients in each group and received intravenous infusion midazolam (Group I), propofol (Group II), dexmedetomidine (Group III) and normal saline (Group IV) as sedation.

\section{RESULTS}

Mean pulse rate reduced in all the four groups up to 90 mins following spinal anaesthesia. No clinical significance was seen at 90 mins, when intergroup comparison was made. The mean systolic and diastolic blood pressure in all the four groups were up to 90 mins. Intergroup comparison was significant $(\mathrm{p}<0.001)$. The onset of sedation was earliest in group II and prolonged in group III as compared to group I. Intergroup comparison is highly significant ( $p<0.001)$. Maximum mean postoperative analgesia was in group III followed by group I and II and IV respectively. Intergroup comparison shows highly significant difference in all groups (p $<0.001$ ). Time taken to reach sedation score 5 was highest in group III followed by group I and II. Intergroup comparison was highly significant.

\section{CONCLUSION}

The onset of sedation was earlier in patient who received IV propofol infusion under spinal anaesthesia as compared to patients who receive IV infusion of midazolam or dexmedetomidine or normal saline. Postoperative analgesia was maximum in patients who receive IV dexmedetomidine infusion as compared to others.

\section{KEYWORDS}

Dexmedetomidine, Propofol, Midazolam, Infusion, Intravenous Sedation.

HOW TO CITE THIS ARTICLE: Mantan K, Pareek A, Jain R, et al. A comparative evaluation of dexmedetomidine, propofol and midazolam for intraoperative sedation in regional anaesthesia. J. Evolution Med. Dent. Sci. 2017;6(75):5387-5392, DOI: $10.14260 /$ Jemds/2017/1168

\section{BACKGROUND \\ Regional anaesthesia is popular and offers several benefits to the patient. The top three from the patient's point of view are staying awake, early family contact and early food intake. ${ }^{5}$ This shows that patients are interested in postoperative landmarks ${ }^{1,2}$ and their importance regarding patient's satisfaction. For the anaesthetist, cardiovascular and respiratory stability, rapid postoperative recovery and preservation of protective airway reflexes are the most}

Financial or Other, Competing Interest: None.

Submission 24-07-2017, Peer Review 06-09-2017,

Acceptance 12-09-2017, Published 18-09-2017.

Corresponding Author:

Dr. Anita Pareek,

4/E/188, J. N. Vyas Colony,

Bikaner, Rajasthan.

E-mail: dranitapareek23@gmail.com

DOI: $10.14260 /$ jemds $/ 2017 / 1168$

\section{(c) $($ ) $\$$}

important advantages of regional anaesthesia. Some drawbacks linked with regional anaesthetic techniques are pain at the puncture site, ${ }^{1}$ fear of needles ${ }^{3}$ and recall of the procedure. ${ }^{4}$ These factors stress the importance of sedation that offers analgesia, anxiolysis and amnesia. Sedation is part of the general management of a patient receiving a regional block and being awake during the whole surgical procedure. The aims include general patient comfort, freedom from specific discomfort and some amnesia for both the block procedure and the surgical operation. Ideal sedative agent should follow for rapid modification of sedation level by modification of dosage (titrable) and should not have depressant effect on cardiovascular and respiratory system.

Midazolam remains the most popular because of its predictable sedative, anxiolytic and amnestic properties irrespective of the route of administration (i.e. oral, topical or parenteral).5,6,7,8 Propofol, IV sedative hypnotic of choice at the present time has been found to be equivalent to 
midazolam for providing adequate sedation and amnesia during regional anaesthesia.9,10,11,12 Dexmedetomidine is a new highly selective and potent alpha-2 adrenoceptor agonist offering sedation and anxiolysis, has analgesic qualities and reduce the stress response to surgery and intensive care procedure.

\section{Aim and Objectives}

To compare dexmedetomidine, propofol and midazolam in intraoperative and postoperative sedation in regional anaesthesia.

To provide better haemodynamic control and better sedation and analgesia in intra- and post-operative period with minimal complications.

To provide safety, comfort and amnesia for both the block and surgical procedure.

\section{MATERIALS AND METHODS}

This randomised, double-blind, controlled trial was conducted on 80 patients in the Department of Anaesthesiology, Sardar Patel Medical College and Associated Group of Hospitals, Bikaner, after taking permission from Institution Research Board. Both male and female patients ranging between the age group of 20 - 60 years belonging to ASA grades I and II, weighing $40-70 \mathrm{~kg}$, scheduled for elective lower limb or lower abdominal surgical procedure which all anticipated to complete within 2 hours under regional anaesthesia were included in selection of patients.

80 adult patients of either sex were included, belonging to ASA grades I and II. They were randomised into 4 groups and efforts were made that the group do not significantly differ with respect to age, weight and height. Sample size was calculated considering two-sided alpha error at 5\% and power of study $80 \%$ with formula $\mathrm{m}=2 \mathrm{c} /$ delta +1 (where $\mathrm{c}=$ 7.9 for power $80 \%$ and delta in standardised effect size derived from prior studies as .9) and was 19.55 (rounded to 20) for each group.

\section{Exclusion Criteria}

Patient with history of allergic reaction to the study drugs; those with significant cardiac, pulmonary, hepatic or renal dysfunction; obese patients ( $>130 \%$ ideal body weight); those with history of chronic use of sedative drugs; full stomach patients, pregnant patients and epileptic patients all were excluded from the study. Pre-anaesthetic checkup.

Pre-anaesthetic checkup was carried out the day before surgery. Routine lab investigations like haemogram, blood sugar, blood urea, serum creatinine, chest x-ray and ECG were done. Standard monitors were applied with noninvasive BP, PR, Heart rate and SPO2 were recorded. The patients were divided into 4 groups of 20 patients in each group.

\section{Group I}

Patients received spinal anaesthesia and IV sedation with midazolam infusion $0.5 \mathrm{mg} / \mathrm{kg} / \mathrm{hr}$.

\section{Group II}

Patients received spinal anaesthesia and IV sedation with propofol $6 \mathrm{mg} / \mathrm{kg} / \mathrm{hr}$.

\section{Group III}

Patients received spinal anaesthesia and IV sedation with dexmedetomidine infusion 0.6 microgram $/ \mathrm{kg} / \mathrm{hr}$.

\section{Group IV}

Patients received spinal anaesthesia and IV infusion of normal saline.

\section{Procedure}

Patients were taken on operating table and baseline BP, Pulse rate, Respiratory rate and SPO2 were recorded. The patients were subsequently shifted to the operating room and were randomly allocated with the help of computer generated random number to receive either propofol $1 \mathrm{mg} / \mathrm{mL}$ (in $5 \%$ dextrose in $50 \mathrm{~mL}$ syringe) or midazolam $0.1 \mathrm{mg} / \mathrm{mL}$ (5\% dextrose in $50 \mathrm{~mL}$ syringe) or dexmedetomidine 2 microgram/mL (in normal saline in $50 \mathrm{~mL}$ syringe) through intravenous infusion. Midazolam was started at an initial infusion rate of $0.5 \mathrm{mg} / \mathrm{kg} / \mathrm{hr}$ or dexmedetomidine started at an infusion rate 0.6 microgram $/ \mathrm{kg} / \mathrm{hr}$ to achieve desired level of sedation score 4 was noted. The infusion rate was lowered or raised in order to maintain sedation score of 4 . After achieving a sedation score of 4 , subarachnoid block was instituted by injecting $2.5 \mathrm{~mL}$ of bupivacaine heavy $0.5 \%$ to achieve adequate sensory block. Sedation score, systolic BP, diastolic BP, HR and SPO2 were recorded 5 mins before intrathecal injection and every 5 mins after intrathecal injection for $30 \mathrm{mins}$, then every $15 \mathrm{mins}$ after up to $90 \mathrm{mins}$ till the surgery continues, then every 2 hours until the patient is under sedation.

Prediction of depth of sedation and anaesthesia was noted by observer's assessment of alertness/sedation score.

\section{Score 5}

Responsiveness- Respond readily to voice with normal tone. Speech- Normal.

Facial Expression- Normal.

Eyes- Clear, No ptosis.

\section{Score 4}

Responsiveness- Respond slowly to voice with normal tone. Speech- Mild slowing.

Facial Expression- Mild relaxation.

Eyes- Marked ptosis (less than half the eye).

\section{Score 3}

Responsiveness- Respond after calling loudly or repeatedly. Speech- Prominent slowing or slurring.

Facial Expression- Marked relaxation.

Eyes- Marked ptosis (half the eye or more).

\section{Score 2}

Responsiveness- Respond after mild prodding or shaking. Speech- Few recognisable words.

Facial Expression- No response.

Eyes- No response.

\section{Score 1}

Responsiveness- Does not respond to mild prodding or shaking.

Speech- No Response.

Facial Expression- No response.

Eyes- No response. 
Score 0

Responsiveness- Does not respond to pain. Speech- No Response.

Facial Expression- No response.

Eyes- No response.
Time to reach postoperative analgesic drug required was noted. Analgesia score was assessed by Visual analgesia score (VAS).

The study data analysed by using Analysis of Variance (ANOVA) and Chi-square test SPSS software 10.0.
All patients were given supplementary oxygen via ventimask at $4 \mathrm{~L} / \mathrm{min}$.

\section{Observations}

Observation/ Statistical Analysis Tables from 1 to 9.

\begin{tabular}{|c|c|c|c|c|c|c|c|c|c|c|}
\hline \multirow{2}{*}{ Mean Pulse } & \multicolumn{2}{|c|}{ Group I } & \multicolumn{2}{|c|}{ Group II } & \multicolumn{2}{|c|}{ Group III } & \multicolumn{2}{|c|}{ Group IV } & \multirow{2}{*}{ CD5\% } & \multirow{2}{*}{$\mathbf{P}$} \\
\hline & Mean & SD & Mean & SD & Mean & SD & Mean & SD & & \\
\hline 0 Minute & 87.80 & 10.58 & 82.30 & 6.22 & 88.75 & 13.09 & 90.70 & 16.58 & 1.738 & 0.166 \\
\hline 5 Minutes & 84.00 & 10.91 & 83.00 & 7.10 & 85.10 & 12.18 & 86.30 & 14.26 & 0.310 & 0.818 \\
\hline 10 Minutes & 81.15 & 11.70 & 79.15 & 8.32 & 83.15 & 12.35 & 85.25 & 14.40 & 0.971 & 0.411 \\
\hline 15 Minutes & 78.70 & 13.52 & 76.63 & 8.29 & 81.50 & 12.34 & 81.65 & 14.17 & 0.743 & 0.530 \\
\hline 20 Minutes & 79.45 & 12.91 & 74.58 & 8.73 & 79.05 & 12.55 & 82.80 & 15.15 & 1.396 & 0.251 \\
\hline 25 Minutes & 79.50 & 12.41 & 73.79 & 10.14 & 77.25 & 11.47 & 80.70 & 15.25 & 1.149 & 0.335 \\
\hline 30 Minutes & 77.10 & 9.01 & 72.11 & 8.01 & 75.90 & 9.56 & 79.05 & 15.58 & 1.000 & 0.398 \\
\hline 45 Minutes & 77.88 & 8.08 & 72.00 & 7.73 & 76.44 & 7.82 & 78.32 & 13.59 & 0.635 & 0.595 \\
\hline 60 Minutes & 75.00 & 3.00 & 71.33 & 6.25 & 74.86 & 8.40 & 75.17 & 9.89 & 3.779 & 0.021 \\
\hline 75 Minutes & 72.75 & 2.50 & 69.67 & 4.66 & 73.00 & 5.00 & 73.00 & 7.00 & 4.937 & 0.014 \\
\hline 90 Minutes & 72.00 & 2.45 & 62.25 & 2.21 & 72.00 & 0.00 & 73.50 & 0.071 & 1.446 & 0.309 \\
\hline 2 Hours & 80.10 & 7.33 & 76.80 & 6.30 & 80.40 & 6.60 & 78.60 & 12.33 & 0.753 & 0.524 \\
\hline 4 Hours & 81.00 & 7.83 & 78.40 & 7.33 & 82.50 & 6.65 & 81.10 & 12.03 & 0.770 & 0.514 \\
\hline 6 Hours & 82.30 & 5.40 & 81.30 & 7.79 & 84.70 & 5.96 & 82.30 & 11.88 & 0.627 & 0.600 \\
\hline 8 Hours & 81.60 & 6.89 & 82.30 & 8.57 & 81.90 & 6.88 & 81.90 & 10.33 & 0.024 & 0.995 \\
\hline 12 Hours & 82.30 & 6.50 & 81.80 & 8.48 & 80.70 & 7.06 & 81.30 & 9.18 & 0.151 & 0.929 \\
\hline 24 Hours & 82.30 & 4.96 & 80.20 & 7.11 & 81.80 & 7.05 & 83.20 & 9.25 & 0.776 & 0.511 \\
\hline
\end{tabular}

Table 1. Showing Mean Pulse Rate at different Time Intervals in all the 4 Groups

\begin{tabular}{|c|c|c|c|c|c|c|c|c|c|c|}
\hline \multirow{2}{*}{$\begin{array}{c}\text { Systolic BP } \\
\text { (mmHg) }\end{array}$} & \multicolumn{2}{|c|}{ Group I } & \multicolumn{2}{|c|}{ Group II } & \multicolumn{2}{|c|}{ Group III } & \multicolumn{2}{|c|}{ Group IV } & \multirow{2}{*}{ CD5\% } & \multirow{2}{*}{$\mathbf{P}$} \\
\hline & Mean & SD & Mean & SD & Mean & SD & Mean & SD & & \\
\hline 0 Minute & 124.65 & 12.58 & 122.50 & 12.22 & 130.85 & 12.89 & 137.85 & 10.95 & 6.420 & 0.001 \\
\hline 5 Minutes & 113.95 & 12.55 & 117.75 & 11.63 & 121.25 & 15.49 & 129.00 & 13.78 & 4.548 & 0.006 \\
\hline 10 Minutes & 110.00 & 12.54 & 111.10 & 13.91 & 116.05 & 17.14 & 128.15 & 15.15 & 6.321 & 0.001 \\
\hline 15 Minutes & 109.20 & 15.37 & 107.00 & 12.65 & 113.50 & 15.81 & 127.65 & 16.52 & 7.379 & $<0.001$ \\
\hline 20 Minutes & 107.25 & 15.88 & 103.58 & 9.23 & 115.05 & 15.10 & 125.80 & 15.78 & 9.299 & $<0.001$ \\
\hline 25 Minutes & 106.70 & 13.27 & 103.32 & 11.56 & 114.70 & 13.78 & 125.25 & 17.15 & 9.401 & $<0.001$ \\
\hline 30 Minutes & 108.53 & 12.92 & 104.78 & 11.45 & 114.35 & 13.32 & 124.16 & 15.94 & 7.282 & $<0.001$ \\
\hline 45 Minutes & 107.00 & 13.23 & 102.27 & 7.20 & 116.53 & 9.96 & 121.80 & 18.17 & 7.468 & $<0.001$ \\
\hline 60 Minutes & 99.40 & 15.13 & 99.44 & 6.09 & 114.00 & 11.27 & 119.40 & 19.31 & 4.288 & 0.013 \\
\hline 75 Minutes & 94.75 & 7.09 & 100.33 & 6.50 & 103.33 & 7.57 & 119.00 & 15.59 & 5.054 & 0.013 \\
\hline 90 Minutes & 95.25 & 4.57 & 103.00 & 4.97 & 98.00 & 0.00 & 122.50 & 17.68 & 5.323 & 0.032 \\
\hline 2 Hours & 112.00 & 8.94 & 115.40 & 7.54 & 115.00 & 7.61 & 118.80 & 7.74 & 2.433 & 0.071 \\
\hline 4 Hours & 118.50 & 10.89 & 119.30 & 5.78 & 122.70 & 10.04 & 125.60 & 12.37 & 2.108 & 0.106 \\
\hline 6 Hours & 123.70 & 8.27 & 122.00 & 7.57 & 123.00 & 8.91 & 128.90 & 9.98 & 2.492 & 0.066 \\
\hline 8 Hours & 122.70 & 10.75 & 121.30 & 9.18 & 125.90 & 8.47 & 125.30 & 6.99 & 1.175 & 0.325 \\
\hline 12 Hour & 125.20 & 10.31 & 120.20 & 7.22 & 123.90 & 9.35 & 125.90 & 7.47 & 1.710 & 0.172 \\
\hline 24 Hours & 124.50 & 9.45 & 119.40 & 6.87 & 120.60 & 7.02 & 125.00 & 9.74 & 2.224 & 0.092 \\
\hline
\end{tabular}

\begin{tabular}{|c|c|c|c|c|c|c|c|c|c|c|}
\hline \multirow{2}{*}{$\begin{array}{c}\text { Diastolic BP } \\
\text { (mmHg) }\end{array}$} & \multicolumn{2}{|c|}{ Group I } & \multicolumn{2}{|c|}{ Group II } & \multicolumn{2}{|c|}{ Group III } & \multicolumn{2}{|c|}{ Group IV } & \multirow{2}{*}{ CD5\% } & \multirow{2}{*}{$\mathbf{P}$} \\
\hline & Mean & SD & Mean & SD & Mean & SD & Mean & SD & & \\
\hline 0 Minute & 82.80 & 8.69 & 80.70 & 6.84 & 84.40 & 10.03 & 84.55 & 8.00 & 0.896 & 0.447 \\
\hline 5 Minutes & 76.35 & 9.19 & 77.40 & 7.92 & 79.50 & 8.49 & 83.85 & 7.42 & 3.214 & 0.028 \\
\hline 10 Minutes & 73.20 & 9.27 & 73.35 & 8.68 & 76.25 & 8.72 & 80.50 & 6.75 & 3.304 & 0.025 \\
\hline 15 Minutes & 71.60 & 10.65 & 68.89 & 9.10 & 74.35 & 8.74 & 79.40 & 8.71 & 4.527 & 0.006 \\
\hline 20 Minutes & 70.00 & 9.02 & 66.63 & 7.80 & 75.90 & 9.14 & 78.70 & 9.91 & 7.251 & $<0.001$ \\
\hline 25 Minutes & 70.30 & 7.08 & 66.42 & 9.47 & 74.30 & 7.95 & 78.65 & 10.34 & 6.989 & $<0.001$ \\
\hline 30 Minutes & 70.05 & 7.34 & 67.00 & 10.57 & 74.95 & 9.11 & 79.70 & 10.33 & 6.716 & $<0.001$ \\
\hline 45 Minutes & 70.29 & 8.76 & 64.20 & 8.01 & 76.12 & 8.07 & 78.69 & 10.54 & 8.208 & $<0.01$ \\
\hline 60 Minutes & 63.60 & 11.08 & 63.11 & 10.01 & 76.29 & 9.34 & 78.67 & 10.34 & 5.561 & 0.004 \\
\hline 75 Minutes & 65.25 & 11.76 & 63.78 & 9.91 & 67.67 & 8.74 & 78.67 & 14.15 & 1.469 & 0.263 \\
\hline 90 Minutes & 64.75 & 6.70 & 61.00 & 7.79 & 68.00 & 0.00 & 82.00 & 15.56 & 2.568 & 0.137 \\
\hline 2 Hours & 76.00 & 5.98 & 77.20 & 5.89 & 77.80 & 6.15 & 79.60 & 6.54 & 1.191 & 0.319 \\
\hline
\end{tabular}




\begin{tabular}{|c|c|c|c|c|c|c|c|c|c|c|}
\hline 4 Hours & 77.40 & 7.95 & 77.00 & 4.66 & 80.40 & 6.34 & 81.50 & 6.80 & 2.294 & 0.085 \\
\hline 6 Hours & 79.50 & 7.31 & 81.40 & 5.59 & 81.90 & 7.41 & 82.80 & 6.82 & 0.834 & 0.479 \\
\hline 8 Hours & 81.40 & 5.15 & 79.90 & 6.60 & 81.30 & 7.85 & 84.60 & 6.09 & 1.873 & 0.141 \\
\hline 12 Hours & 82.60 & 6.36 & 80.70 & 4.78 & 80.50 & 5.91 & 83.30 & 5.78 & 1.173 & 0.326 \\
\hline 24 Hours & 82.60 & 5.66 & 80.30 & 3.85 & 82.70 & 9.32 & 84.00 & 5.66 & 1.142 & 0.338 \\
\hline
\end{tabular}

\begin{tabular}{|c|c|c|c|c|c|c|c|c|c|c|}
\hline \multirow{2}{*}{$\begin{array}{c}\text { Respiratory } \\
\text { Rate (min) }\end{array}$} & \multicolumn{2}{|c|}{ Group I } & \multicolumn{2}{c|}{ Group II } & \multicolumn{2}{c|}{ Group III } & \multicolumn{2}{c|}{ Group IV } & \multirow{2}{*}{ CD5\% } & \multirow{2}{*}{ p } \\
\hline & Mean & SD & Mean & SD & Mean & SD & Mean & SD & & 0.729 \\
\hline 5 Minutes & 15.65 & 0.75 & 15.65 & 0.67 & 15.95 & 60.60 & 15.70 & 1.56 & 0.434 & 0.729 \\
\hline 10 Minutes & 15.05 & 0.69 & 15.00 & 0.86 & 15.40 & 0.94 & 15.95 & 1.82 & 2.337 & 0.054 \\
\hline 15 Minutes & 14.80 & 1.01 & 14.84 & 0.96 & 14.95 & 0.76 & 15.55 & 1.43 & 2.120 & 0.105 \\
\hline 20 Minutes & 14.85 & 0.99 & 14.74 & 0.99 & 15.10 & 0.79 & 15.55 & 1.27 & 2.437 & 0.071 \\
\hline 25 Minutes & 15.00 & 0.79 & 14.58 & 0.90 & 14.90 & 0.79 & 15.35 & 1.27 & 2.140 & 0.102 \\
\hline 30 Minutes & 14.68 & 0.95 & 14.72 & 0.75 & 14.75 & 0.72 & 15.35 & 1.31 & 2.122 & 0.105 \\
\hline 45 Minutes & 14.56 & 0.63 & 14.47 & 1.06 & 14.88 & 0.86 & 15.26 & 1.19 & 2.391 & 0.077 \\
\hline 60 Minutes & 14.20 & 0.45 & 14.33 & 1.12 & 14.43 & 0.53 & 15.83 & 1.95 & 2.444 & 0.061 \\
\hline 75 Minutes & 13.50 & 1.00 & 14.33 & 0.71 & 14.33 & 0.58 & 13.67 & 1.52 & 1.060 & 0.395 \\
\hline 90 Minutes & 13.50 & 1.00 & 14.25 & 0.50 & 14.00 & 0.00 & 14.50 & 0.71 & 0.961 & 0.462 \\
\hline 2 Hours & 15.90 & 0.31 & 15.85 & 0.50 & 15.75 & 0.44 & 16.10 & 1.21 & 0.869 & 0.461 \\
\hline 4 Hours & 15.70 & 0.73 & 15.90 & 1.29 & 15.75 & 0.64 & 16.05 & 1.19 & 0.495 & 0.687 \\
\hline 6 Hours & 15.95 & 1.50 & 16.70 & 1.45 & 16.80 & 1.82 & 17.20 & 1.54 & 2.161 & 0.100 \\
\hline 8 Hours & 16.05 & 1.15 & 17.05 & 2.50 & 17.10 & 2.07 & 17.55 & 1.70 & 2.170 & 0.098 \\
\hline 12 Hours & 16.15 & 0.93 & 16.85 & 1.14 & 17.25 & 2.81 & 17.60 & 1.73 & 2.377 & 0.076 \\
\hline 24 Hours & 16.30 & 1.08 & 17.00 & 1.26 & 16.25 & 1.45 & 16.95 & 1.43 & 1.906 & 0.136 \\
\hline
\end{tabular}

\begin{tabular}{|c|c|c|c|c|c|c|c|c|c|c|}
\hline \multirow{2}{*}{$\mathrm{SPO}^{2}(\%)$} & \multicolumn{2}{|c|}{ Group I } & \multicolumn{2}{|c|}{ Group II } & \multicolumn{2}{|c|}{ Group III } & \multicolumn{2}{|c|}{ Group IV } & \multirow{2}{*}{ CD5\% } & \multirow{2}{*}{$\mathbf{P}$} \\
\hline & Mean & SD & Mean & SD & Mean & SD & Mean & SD & & \\
\hline 5 Minutes & 99.10 & 1.07 & 99.20 & 1.06 & 99.05 & 0.94 & 99.65 & 0.75 & 1.617 & 0.192 \\
\hline 10 Minutes & 98.85 & 1.04 & 98.95 & 1.36 & 98.65 & 0.99 & 99.55 & 0.83 & 2.621 & 0.057 \\
\hline 15 Minutes & 98.85 & 1.04 & 99.42 & 0.96 & 99.15 & 0.93 & 99.60 & 0.75 & 2.481 & 0.067 \\
\hline 20 Minutes & 99.15 & 0.93 & 99.26 & 1.59 & 99.45 & 0.99 & 99.80 & 0.70 & 1.336 & 0.269 \\
\hline 25 Minutes & 99.50 & 0.69 & 99.16 & 1.07 & 99.55 & 0.60 & 99.65 & 0.75 & 1.406 & 0.248 \\
\hline 30 Minutes & 99.47 & 0.77 & 99.17 & 0.99 & 99.60 & 0.60 & 99.70 & 0.80 & 1.584 & 0.201 \\
\hline 45 Minutes & 99.35 & 0.93 & 99.13 & 0.92 & 99.65 & 0.79 & 99.68 & 0.67 & 1.633 & 0.190 \\
\hline 60 Minutes & 99.00 & 1.22 & 98.78 & 1.20 & 99.00 & 1.15 & 99.50 & 1.00 & 0.783 & 0.513 \\
\hline 75 Minutes & 99.00 & 1.15 & 98.50 & 1.31 & 98.67 & 0.58 & 100.00 & 0.00 & 1.431 & 0.276 \\
\hline 90 Minutes & 99.25 & 0.96 & 99.25 & 0.96 & 99.00 & 0.00 & 99.50 & 0.71 & 0.071 & 0.974 \\
\hline 2 Hours & 97.20 & 0.70 & 97.70 & 1.22 & 97.80 & 1.28 & 97.25 & 0.44 & 1.974 & 0.125 \\
\hline 4 Hours & 97.15 & 0.37 & 97.65 & 1.09 & 97.35 & 0.81 & 97.45 & 0.51 & 1.546 & 0.209 \\
\hline 6 Hours & 97.20 & 0.41 & 97.65 & 1.09 & 97.40 & 0.88 & 97.45 & 0.51 & 1.141 & 0.338 \\
\hline 8 Hours & 97.25 & 0.44 & 97.40 & 0.50 & 97.30 & 0.47 & 97.60 & 0.60 & 1.863 & 0.143 \\
\hline 12 Hours & 97.25 & 0.44 & 97.45 & 0.76 & 97.10 & 0.72 & 97.40 & 0.50 & 1.297 & 0.282 \\
\hline 24 Hours & 97.30 & 0.47 & 97.80 & 1.06 & 97.30 & 0.47 & 97.45 & 0.60 & 2.313 & 0.083 \\
\hline
\end{tabular}

\begin{tabular}{|c|c|c|c|c|c|c|c|c|}
\hline \multirow{2}{*}{$\begin{array}{c}\text { Onset of } \\
\text { Sedation } \\
\text { (Minutes) }\end{array}$} & \multicolumn{2}{|c|}{ Group I } & \multicolumn{2}{|c|}{ Group II } & \multicolumn{2}{|c|}{ Group III } & \multicolumn{2}{|c|}{ Group IV } \\
\hline & No. & $\%$ & No. & $\%$ & No. & $\%$ & No. & $\%$ \\
\hline $5-7$ & 2 & 10 & 11 & 55 & 0 & - & - & - \\
\hline $8-10$ & 15 & 75 & 8 & 40 & 0 & - & - & - \\
\hline 11-14 & 3 & 15.0 & 1 & 5 & 2 & 10.0 & - & - \\
\hline $15-20$ & 0 & - & 0 & - & 16 & 80 & - & - \\
\hline$>20$ & 0 & - & 0 & - & 2 & 10.0 & - & - \\
\hline Total & 20 & 100 & 20 & 100 & 20 & 100 & - & - \\
\hline Mean & \multicolumn{2}{|c|}{9.00} & \multicolumn{2}{|c|}{7.15} & \multicolumn{2}{|c|}{18.25} & - & - \\
\hline SD & \multicolumn{2}{|c|}{1.72} & \multicolumn{2}{|c|}{1.66} & \multicolumn{2}{|c|}{2.12} & - & - \\
\hline & & & & & & \\
\hline \multicolumn{5}{|c|}{ Group I vs II } & \multicolumn{2}{|c|}{3.461} & \multicolumn{2}{|c|}{$=0.001$} \\
\hline \multicolumn{5}{|c|}{ Group I vs III } & \multicolumn{2}{|c|}{15.145} & \multicolumn{2}{|c|}{$<0.001$} \\
\hline \multicolumn{5}{|c|}{ Group II vs III } & \multicolumn{2}{|c|}{18.399} & \multicolumn{2}{|c|}{$<0.001$} \\
\hline \multicolumn{9}{|c|}{$\begin{array}{l}\text { Table 6. Showing Distribution of Cases according } \\
\text { to Onset of Sedation in all the } 4 \text { Study Groups }\end{array}$} \\
\hline
\end{tabular}

\begin{tabular}{|c|c|c|c|c|c|c|c|c|c|c|}
\hline \multirow{2}{*}{ Complications } & \multicolumn{2}{|c|}{\begin{tabular}{|l|} 
Group I \\
\end{tabular}} & \multicolumn{2}{|c|}{ Group II } & \multicolumn{2}{|c|}{ Group III } & \multicolumn{2}{|c|}{ Group IV } & \multicolumn{2}{|c|}{ Total } \\
\hline & \begin{tabular}{|l|} 
No. \\
\end{tabular} & $\%$ & \begin{tabular}{|l|} 
No. \\
\end{tabular} & $\%$ & \begin{tabular}{|l|} 
No. \\
\end{tabular} & $\%$ & No. & $\%$ & No. & $\%$ \\
\hline Nil & 15 & 75 & 16 & 80 & 15 & 75 & 16 & 80 & 62 & 77.5 \\
\hline Hypotension & 0 & - & 2 & 10 & 0 & - & 0 & - & 2 & 2.5 \\
\hline Bradycardia & 1 & 5 & 0 & - & 1 & 5 & 0 & - & 2 & 2.5 \\
\hline Nausea & 1 & 5 & 0 & - & 1 & 5 & 1 & 5 & 3 & 3.7 \\
\hline Vomiting & 1 & 5 & 0 & - & 1 & 5 & 1 & 5 & 3 & 3.7 \\
\hline $\begin{array}{c}\text { Urinary } \\
\text { Retention }\end{array}$ & 2 & 10 & 2 & 10 & 2 & 10 & 2 & 10 & 8 & 10 \\
\hline Total & 20 & 100 & 20 & 100 & 20 & 100 & 20 & 100 & 80 & 100 \\
\hline $\mathrm{X} 2$ & \multicolumn{8}{|c|}{14.486} & & \\
\hline $\mathrm{P}$ & \multicolumn{8}{|c|}{0.106} & & \\
\hline \multicolumn{11}{|c|}{ Table 7. Showing Distribution of Cases } \\
\hline
\end{tabular}




\begin{tabular}{|c|c|c|c|c|c|c|c|c|}
\hline \multirow{2}{*}{$\begin{array}{c}\text { Postoperative } \\
\text { Analgesia } \\
\text { (Hours) }\end{array}$} & \multicolumn{2}{|c|}{ Group I } & \multicolumn{2}{|c|}{ Group II } & \multicolumn{2}{|c|}{ Group III } & \multicolumn{2}{|c|}{ Group IV } \\
\hline & No. & $\%$ & No. & $\%$ & No. & $\%$ & No. & $\%$ \\
\hline $0-2$ & 5 & 25 & 15 & 75 & 0 & - & 20 & 100 \\
\hline $3-4$ & 15 & 75 & 5 & 25 & 3 & 15 & 0 & - \\
\hline $5-6$ & 0 & - & 0 & - & 7 & 35 & 0 & - \\
\hline$>6$ & 0 & - & 0 & - & 10 & 50 & 0 & - \\
\hline Total & 20 & 100 & 20 & 100 & 20 & 100 & 20 & 100 \\
\hline Mean & \multicolumn{2}{|c|}{\begin{tabular}{l|}
3.15 \\
\end{tabular}} & \multicolumn{2}{|c|}{\begin{tabular}{|l|}
1.93 \\
\end{tabular}} & \multicolumn{2}{|c|}{$\begin{array}{l}1 \\
6.55\end{array}$} & \multicolumn{2}{|c|}{1.25} \\
\hline SD & \multicolumn{2}{|c|}{0.86} & \multicolumn{2}{|c|}{0.96} & \multicolumn{2}{|c|}{1.76} & \multicolumn{2}{|c|}{0.62} \\
\hline & & & & & \multicolumn{2}{|c|}{$\mathrm{t}$} & \multicolumn{2}{|c|}{$\mathrm{p}$} \\
\hline \multicolumn{3}{|c|}{ Group I vs II } & & & \multicolumn{2}{|c|}{4.242} & \multicolumn{2}{|c|}{$<0.001$} \\
\hline \multicolumn{3}{|c|}{ Group I vs III } & & & \multicolumn{2}{|c|}{7.757} & \multicolumn{2}{|c|}{$<0.001$} \\
\hline \multicolumn{3}{|c|}{ Group I vs IV } & & & \multicolumn{2}{|c|}{8.025} & \multicolumn{2}{|c|}{$<0.001$} \\
\hline \multicolumn{3}{|c|}{ Group II vs III } & & & \multicolumn{2}{|c|}{10.302} & \multicolumn{2}{|c|}{$<0.001$} \\
\hline \multicolumn{3}{|c|}{ Group II vs IV } & & & \multicolumn{2}{|c|}{2.638} & \multicolumn{2}{|c|}{0.012} \\
\hline \multicolumn{3}{|c|}{ Group III vs IV } & & & \multicolumn{2}{|c|}{12.698} & \multicolumn{2}{|c|}{$<0.001$} \\
\hline
\end{tabular}

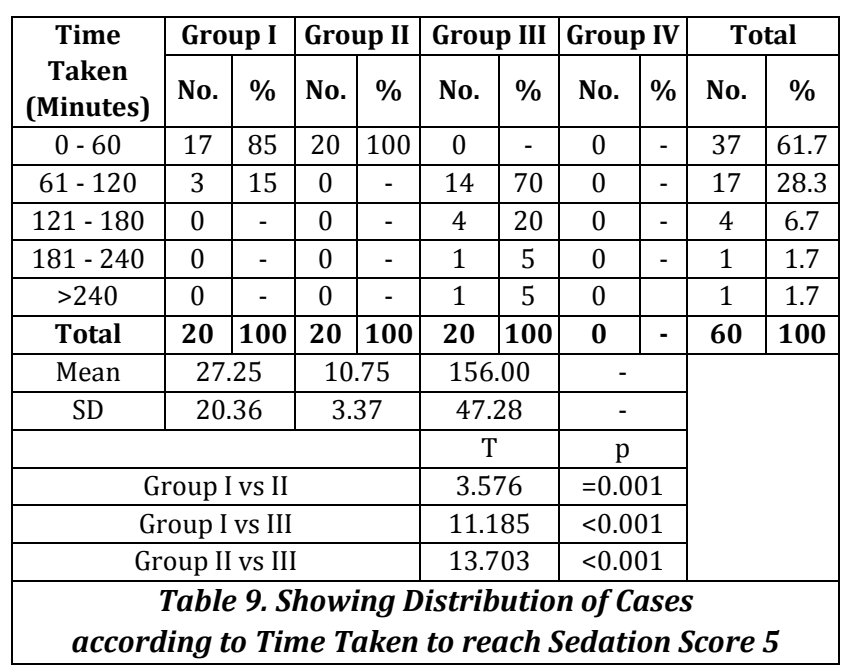

\section{RESULTS}

There were no significant differences between the four groups with regard to age, weight and gender of the patients. We observed Pulse rate, Systolic blood pressure, Diastolic blood pressure and $\mathrm{SPO}_{2}$ to various extent. There was gradual fall in the pulse rate upto 45 mins following spinal anaesthesia in each group. No significant change in pulse was seen when intergroup comparison was made between four groups till 90 mins. Comparison of mean systolic blood pressure at various intervals in 4 groups showed that the groups were statistically highly significant $(p<0.001)$ till 45 minutes. After 45 minutes the difference was statistically significant $(\mathrm{p}<0.05)$. It was highly significant in group propofol till 45 minutes than other groups. The intergroup comparison of mean diastolic blood pressure showed that the groups were statistically significant $(\mathrm{p}<0.05)$ till 15 mins and after 15 mins till 45 mins it was highly significant. No clinical significant changes in $\mathrm{SPO}_{2}$ was seen when intergroup comparison was made between 4 groups.

We also observed time taken for onset of sedation, which was earliest in propofol group and prolonged in dexmedetomidine group as compared to midazolam group. The statistical intergroup comparison shows the highly significant difference in all the groups $(\mathrm{p}<0.001)$.
Hypotension was seen in 2 patients in group propofol. Bradycardia was seen in 1 patient in each midazolam and propofol group, while nausea and vomiting was present in 1 case in each group of midazolam, dexmedetomidine and control group, while urinary retention was seen in 2 cases in all the 4 groups. This difference was statistically insignificant $(\mathrm{p}>0.05)$.

Maximum mean postoperative analgesia was in group dexmedetomidine followed by group midazolam, propofol and control respectively. Intergroup comparison was highly significant in all groups $(\mathrm{p}<0.001)$.

Time taken to reach sedation score 5 was lowest in group II propofol (10.75 +/- 3.37 minutes) and maximum in group III dexmedetomidine $(156.00+/-47.28$ minutes $)$. Time taken in group I midazolam (27.25 +/- 20.36 minutes).

\section{DISCUSSION}

The use of spinal anaesthesia is often limited by the unwillingness of the patient to remain awake during surgery. Supplemental sedation with an intravenous agent is often required to allay fear and anxiety in patients subjected to spinal anaesthesia. Most widely used technique for administering sedation in regional anaesthesia is the intermittent intravenous bolus dose technique. This technique is associated with peaks and troughs in plasma concentration producing significant side effects and delayed recovery. Continuous infusions have been proved to produce lesser side effects, faster recovery and easy controllability over the desired level of sedation.

Mean pulse rate reduced in all the 4 groups upto 90 minutes. Magalhaes et $\mathrm{al}^{13}$ found that were no statistical significant difference between dexmedetomidine and midazolam groups in heart rate variation. Celik et al ${ }^{14}$ also found that in comparison to propofol, dexmedetomidine achieved more stable haemodynamic parameters. Similar results were observed in our study.

Mean systolic blood pressure reduced in all 4 groups upto 90 minutes. Intergroup comparison significant ( $<<0.001)$. The mean diastolic blood pressure also reduced in all 4 groups upto 75 minutes following spinal anaesthesia, which was statistically highly significant $(p<0.001)$. The study correlates well with Arain and Ebert, ${ }^{15}$ Magalhaes et al ${ }^{13}$ and Celik et al ${ }^{14}$ and Liang et al. ${ }^{16}$

Changes in $\mathrm{SPO}_{2}$ were clinically insignificant among all groups ( $p>0.05$ ). Our study correlates with Patki and Shelgaonkar. ${ }^{17}$

The mean time for onset of sedation as assessed by OASS score was found to be $9.00+/-1.72 \mathrm{mins}, 7.15+/-1.66 \mathrm{mins}$ and $18.25+/-2.12$ mins in group I, II and III. Studies by Arain and Ebert, 15 Patki and Shelgaonkar ${ }^{17}$ and Liang et al ${ }^{16}$ correlates well with our study.

No significant complications were seen among groups. Our study favours studies by White and Smith ${ }^{18}$ and Patki and Shelgaonkar. ${ }^{17}$

Maximum mean postoperative analgesia was highest in group III (Dexmedetomidine) followed by group I (midazolam) and group II (propofol) and group IV (control). Celik et $\mathrm{al}^{14}$ found that time for first analgesic requirement was significantly longer in group dexmedetomidine as compared to midazolam. Kaya et $\mathrm{al}^{19}$ concluded that dexmedetomidine increased the time for first request for 
postoperative analgesia compared with midazolam and saline. Our study correlates with above authors.

Time taken to reach complete awakening (Sedation Score 5) was highest in dexmedetomidine II group followed by midazolam I group and propofol II group. Intergroup comparison was significant. Our study corresponds with Yao et al. ${ }^{20}$

\section{CONCLUSION}

Operative condition was good and comparable with good haemodynamic stability in all 4 groups. Midazolam is highly suitable with significantly less pain on injection, lower incidence of postoperative venoirritation and its lower cost. Propofol is highly suitable as a sedative for spinal anaesthesia in spite of injection pain. It is superior to midazolam and dexmedetomidine, because of significantly early onset of sedation and shorter recovery time. Dexmedetomidine achieved similar level of sedation to midazolam and propofol in lower limb surgery under spinal anaesthesia. Patients sedated with dexmedetomidine could be easily aroused to cooperate with procedure without showing irritation. Adverse side effects of dexmedetomidine, midazolam and propofol are avoided by the slow infusion of drugs.

From the results of our clinical study we concluded that all the three drugs provide good sedation, stable haemodynamics, no respiratory depression and alertness with good cooperation. Midazolam is the drug of choice due to its cost benefit; propofol is preferred during ambulatory surgery under regional anaesthesia and dexmedetomidine is used as first option by the additional analgesic property.

\section{REFERENCES}

[1] Lonsdale M, Hutchison GL. Patient's desire for information about anaesthesia. Scottish and Canadian attitudes. Anaesthesia 1991;46(5):410-2.

[2] Asehnoune K, Albaladejo P, Smail N, et al. Information and anaesthesia: what does the patient desire? ANN Fr Anesth Reanim 2000;19(8):577-81.

[3] Gajraj NM, Sharma SK, Souter AJ, et al. A survey of obstetric patients who refuse regional anaesthesia. Anaesthesia 1995;50(8):740-1.

[4] Marcario A, Weinger M, Carney S, et al. Which clinical anaesthesia outcomes are important to avoid? The persrective of patients. Anesth Analg 1999;89(3):6528.

[5] Vlymen VJJ, Sa Rego MM, White PF. Benzodiazepine premedication: can it improve outcome in patients undergoing breast biopsy procedures? Anaesthesiology 1999;90(3):740-7.

[6] Al-Rakaf H, Bello LL, Turkostani A, et al. Intra-nasal midazolam in conscious sedation of young paediatric dental patients. Int J Paediatr Dent 2001;11(1):33-40.

[7] Cote CJ, Cohen IT, Suresh S, et al. A comparison of three doses of a commercially prepared oral midazolam syrup in children. Anesth Analg 2002;94(1):37-43.

[8] Habib NE, Mandour NM, Balmer HG. Effect of midazolam on anxiety level and pain perception in cataract surgery with topical anaesthesia. J Cataract Refract Surg 2004;30(2):437-43.

[9] White PF, Negus JB. Sedative infusions during local and regional anesthesia: a comparison of midazolam and propofol. J Clin Anesth 1991;3(1):32-9.

[10] Ferrari LR, Donlon JV. A comparison of propofol, midazolam and methohexital for sedation during retrobulbar and peribulbar block. J Clin Anesth 1992;4(2):93-6.

[11] Pratila MG, Fischer ME, Alagesan R, et al. Propofol vs midazolam for monitored sedation: a comparison of intraoperative and recovery parameters. J Clin Anesth 1993;5(4):268-74.

[12] Cillo JE. Propofol anaesthesia for outpatient for oral and maxillofacial surgery. Oral Surg Oral Med Oral Pathol Oral Radiol Endod 1999;87(5):530-8.

[13] Magalhaes E, Ladeira LC, Govera CS, et al. Intravenous dexmedetomidine for sedation does not interfere with sensory and motor block duration during spinal anaesthesia. Rev Bras Anestesiol 2006;56(1):1-7.

[14] Celik M, Koltka N, Cevik B, et al. Intraoperative sedation during epidural anesthesia: dexmedetomidine vs midazolam. The Internet J Anesthesiology 2008;17(2):27.

[15] Arain SR, Ebert TJ. The efficacy, side effects, and recovery characteristics of dexmedetomidine versus propofol when used for intraoperative sedation. Anesth Analg 2002;95(2):461-6.

[16] Liang Y, Gu M, Wang S, et al. A comparison of dexmedetomidine and midazolam for sedation in gynaecologic surgery under epidural anaesthesia. J Curr Surg 2011;1(1):12-8.

[17] Patki A, Shelgaonkar VC. A comparison of equisedative infusions of propofol and midazolam for conscious sedation during spinal anaesthesia-a prospective randomised study. J Anaesthesiol Clin Pharmacol 2011;27 (1):47-53.

[18] White PE, Smith I. Use of sedation techniques during local and regional anaesthesia. Can J Anaesth 1995;42(1):38-54.

[19] Kaya FN, Yavascaoglu B, Turker G, et al. Intravenous dexmedetomidine, but not midazolam, prolongs bupivacaine spinal anesthesia. Can J Anaesth 2010;57(1):39-45.

[20] Yao L, Zhou XM, Zhao JJ. The role of dexmedetomidine in treatment of serious patients in intensive care unit. Zhongguo Wei Zhong Bing Ji Jiu Yi Xue 2010;22(10):632-4. 